\title{
Effect of large mechanical stress on the magnetic properties of embedded Fe nanoparticles
}

\author{
Srinivasa Saranu ${ }^{1}$, Sören Selve ${ }^{2}$, Ute Kaiser ${ }^{2}$, Luyang Han ${ }^{3}$, Ulf Wiedwald ${ }^{3}$, \\ Paul Ziemann ${ }^{3}$ and Ulrich Herr ${ }^{*} 1$
}

\section{Full Research Paper}

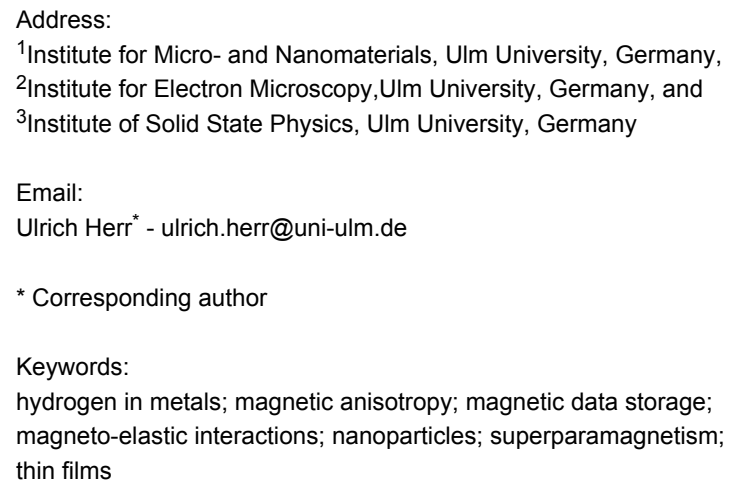

${ }^{1}$ Institute for Micro- and Nanomaterials, Ulm University, Germany,

${ }^{2}$ Institute for Electron Microscopy,Ulm University, Germany, and

${ }^{3}$ Institute of Solid State Physics, Ulm University, Germany

Email:

Ulrich Herr* - ulrich.herr@uni-ulm.de

* Corresponding author

Keywords:

hydrogen in metals; magnetic anisotropy; magnetic data storage; magneto-elastic interactions; nanoparticles; superparamagnetism; thin films

Open Access

Beilstein J. Nanotechnol. 2011, 2, 268-275.

doi:10.3762/bjnano.2.31

Received: 22 February 2011

Accepted: 17 May 2011

Published: 01 June 2011

This article is part of the Thematic Series "Properties and applications of magnetic nanoparticles".

Associate Editor: P. Leiderer

(C) 2011 Saranu et al; licensee Beilstein-Institut.

License and terms: see end of document.

\begin{abstract}
Magnetic nanoparticles are promising candidates for next generation high density magnetic data storage devices. Data storage requires precise control of the magnetic properties of materials, in which the magnetic anisotropy plays a dominant role. Since the total magneto-crystalline anisotropy energy scales with the particle volume, the storage density in media composed of individual nanoparticles is limited by the onset of superparamagnetism. One solution to overcome this limitation is the use of materials with extremely large magneto-crystalline anisotropy. In this article, we follow an alternative approach by using magneto-elastic interactions to tailor the total effective magnetic anisotropy of the nanoparticles. By applying large biaxial stress to nanoparticles embedded in a non-magnetic film, it is demonstrated that a significant modification of the magnetic properties can be achieved. The stress is applied to the nanoparticles through expansion of the substrate during hydrogen loading. Experimental evidence for stress induced magnetic effects is presented based on temperature-dependent magnetization curves of superparamagnetic Fe particles. The results show the potential of the approach for adjusting the magnetic properties of nanoparticles, which is essential for application in future data storage media.
\end{abstract}

\section{Introduction}

Magnetic data storage has been an integral part of computer system technology for many decades and this will most probably remain so in the near future. Over the years, the basic technology of the hard disc, which allows access to magnetic information stored as individual data bits in a magnetic thin film, has been improved without changing the basic concept of moving a read/write head over a rotating disk surface. The impressive advancement in storage density (usually measured in bits per square inch of disc surface) has been achieved by successive reductions of the bit size leading to higher total 
capacities. This development is associated with the introduction of new technologies, such as the magneto-resistive read heads first based on the anisotropic magneto-resistance (AMR) and later on the giant magneto-resistance (GMR) and tunnel magneto-resistance (TMR) effect. In conventional magnetic thin films, each bit comprises a large number of magnetic grains, which are coupled by dipolar interactions and, to some extent, by inter granular exchange coupling. The necessity for incorporation of many grains in each bit arises from the requirement for a sufficiently large signal-to-noise ratio. A further increase of the storage density would require a reduction of the size of individual grains.

The magnetization behavior of small particles has been a topic of interest for many years [1,2]. For particle diameters less than a critical size, a single domain state is expected. In such a case, the magnetization of the particle can be represented by one single magnetic moment which adjusts its direction under the influence of local anisotropies, such as the magneto-crystalline anisotropy field, and external fields. In addition, thermal fluctuations may lead to instability of the magnetization over time, as described by Néel [2] and Brown [3]. If the total anisotropy energy $K_{\text {eff }} \cdot V$ per grain approaches a lower limit of $K_{\text {eff }} V \approx 50-60 k_{\mathrm{B}} T$, the magnetization will switch in an uncontrolled way within a period of 10 years which is generally considered as not acceptable for data storage applications; here, $K_{\text {eff }}$ is the effective anisotropy energy density, $V$ is the volume of the particle and $k_{\mathrm{B}} T$ the thermal energy. The loss of stability can, in principle, be avoided by the use of materials with high coercivity [4], such as chemically ordered FePt or CoPt alloys. However, the use of such materials is limited by the achievable magnetic field of the write head. Patterned media have been discussed as another possible solution, where the data bits are stored in single grains arranged in a regular manner. This would reduce the noise contribution from the irregular domain (bit) boundaries. Spontaneous self-organization of magnetic nanoparticles, as demonstrated first by Sun and co-workers [5] and subsequently by applying micellar preparation techniques [6], has opened up new possibilities for generating this type of media. Another approach for stabilization of the magnetization in small particles is the coupling to an antiferromagnet $[7,8]$. This leads to an increase in the coercivity and additionally, to an exchange bias field, which may shift the magnetization curves along the field axis. This approach is similar to the method used for the pinning of the magnetization of the reference layer in spin valve sensors. In this context, it should be noted that magnetic nanoparticles also have applications in other fields, such as medical treatment, diagnostics and imaging [9].

A precise control of the magnetic anisotropy energy is most important for the design of future magnetic data storage media.
The total effective magnetic anisotropy $K_{\text {eff }}$ is a superposition of contributions from magneto-crystalline $\left(K_{\mathrm{mc}}\right)$, shape $\left(K_{\text {shape }}\right)$, interface $\left(K_{\text {int }}\right)$ and magneto-elastic $\left(K_{\mathrm{me}}\right)$ energies:

$$
K_{\text {eff }}=K_{\text {mc }}+K_{\text {shape }}+K_{\text {int }}+K_{\text {me }}
$$

The values of $K_{\mathrm{mc}}$ range from typically $10^{4}$ to several times $10^{5} \mathrm{~J} / \mathrm{m}^{3}$ for $\mathrm{Fe}, \mathrm{Ni}$ and $\mathrm{Co}$, and up to $6 \cdot 10^{6} \mathrm{~J} / \mathrm{m}^{3}$ for $\mathrm{FePt}$ at ambient temperature [4]. The maximum of $K_{\text {shape }}$ is given by $1 / 2 \cdot \mu_{0} \cdot M_{\mathrm{s}}^{2}$, which can also reach values around $10^{6} \mathrm{~J} / \mathrm{m}^{3}$ for typical saturation magnetization $M_{\mathrm{S}}$ values of about $10^{6} \mathrm{~A} / \mathrm{m}$. Since the contribution from $K_{\text {int }}$ depends on the density of interfaces, it reaches comparable values only when the layer thickness or multilayer periodicities are set in the range of $1 \mathrm{~nm}$. The contribution of $K_{\mathrm{me}}$ may be estimated for isotropic materials by $K_{\mathrm{me}}=3 / 2 \cdot \lambda \cdot \sigma$, where $\lambda$ is the magnetostriction constant and $\sigma$ is the mechanical stress. The value of $\lambda$ varies from typical values of $10^{-4}-10^{-5}$ for most materials up to $10^{-3}$ for some rare earth alloys. To be comparable with the other contributions to the effective anisotropy, stresses in the GPa $\left(10^{9} \mathrm{~Pa}\right)$ range would be required. However, in thin films and other nanostructured materials plastic deformation by dislocation glide is constrained by the presence of surfaces and interfaces. Therefore, large elastic stresses may be present in these materials. In such systems, $K_{\text {me }}$ may contribute significantly to $K_{\text {eff. It has }}$ recently been demonstrated that thin $\mathrm{CoFe}$ and $\mathrm{Ni}$ films subjected to large biaxial stresses show variations of $K_{\text {eff }}$ of up to $50 \%$ [10]. Even larger modifications up to $100 \%$ have been achieved in $\mathrm{Co} / \mathrm{Pd}$ multilayers with perpendicular anisotropy [11]. In these materials, which are interesting as potential perpendicular recording media, the interface anisotropy energy may exceed the shape anisotropy for short multilayer periodicities and cause the magnetization to align perpendicular to the plane of the film in the absence of an external field. The modification of the magnetic anisotropy by large lattice distortions and the generation of additional perpendicular anisotropies has also been demonstrated in the case of CoFe alloys, which show large increases in magnetic anisotropy when subjected to tetragonal distortions by incorporation of the material into $\mathrm{CoFe} / \mathrm{Pt}$ superlattices [12] or growth on $\operatorname{Pd}(001)$ surfaces [13].

In the present contribution, we extend the investigations of the stress effect on the magnetic anisotropy to the study of $\mathrm{Fe}$ nanoparticles embedded in a nonmagnetic film. If the particles are spherical and do not experience a strong dipolar interaction, the value of $K_{\text {shape }}$ should be very low. Bulk Fe has a $K_{\mathrm{mc}}$ of only about $5 \cdot 10^{4} \mathrm{~J} / \mathrm{m}^{3}$, so that contributions from $K_{\text {me }}$ should modify the magnetic behaviour of the nanoparticles even at moderate stress values. We first introduce the experimental method for applying stress and provide evidence for the pres- 
ence of large biaxial stresses. Then we present results from SQUID magnetometry of Fe nanoparticles in the stressed and stress-free state for the same sample over a range of temperatures.

\section{Results and Discussion Deposition and structure of the Fe nanoparticles}

The Fe nanoparticles used in this study were prepared by plasma-assisted gas phase condensation [14]. This method allows deposition of both, elemental [15] and alloy clusters with rather narrow size distributions. By combination with a film deposition technique, in situ embedding of the clusters is possible. This can not only be used to protect the clusters from oxidation, but also to yield new functionalities such as those exhibited in granular giant magneto-resistance (GMR) systems $[16,17]$ or the introduction of exchange bias effects in nanoparticle systems [18]. Although the arrangement of the deposited clusters on the substrate surface is generally random, it has been recently demonstrated that a self-assembly of the clusters is possible by deposition on a polymer film which subsequently coats and separates the particles [19]. The particle size distribution generated under these conditions was examined by scanning electron microscopy (SEM) and atomic force microscopy (AFM). Figure 1 shows a representative sample of Fe nanoparticles deposited on a silicon wafer. The particle diameters follow a log-normal distribution, typical for the gas condensation technique [20], with a mean size of $13.3 \mathrm{~nm}$ and distribution width $\sigma=2.6 \mathrm{~nm}$. For structural investigations by transmission electron microscopy (TEM), the particles were covered in situ with a thin $\mathrm{SiO}_{x}$ layer to avoid oxidation during the transfer to the TEM. Figure 2 shows a representative TEM image of Fe particles. Different sets of lattice planes can be observed in the particle, proving that the particles themselves consist of subparticles with different lattice orientations. This indicates that the particles are most likely formed by agglomeration of small primary clusters during transport in the inert carrier gas. Figure 3 shows the electron diffraction pattern obtained from a number of such particles. The superposition of the diffraction spots leads to Debye-Scherrer rings which can all be attributed to Bragg reflections from bcc Fe, proving that the Fe nanoparticles crystallize in the bcc phase.

\section{Embedding and stress application procedure}

In this work, stress was applied to the nanoparticles by increasing the volume of the substrate on which they have been deposited through loading it with hydrogen after the deposition process. This method has previously been successfully applied in the case of thin films of $\mathrm{Ni}, \mathrm{FeCo}$ and $\mathrm{Co} / \mathrm{Pd}[10,11,21]$. Ta foils (thickness $200 \mu \mathrm{m}$ ) were used as substrates, which can be loaded with hydrogen up to concentrations of several $10 \%$ at

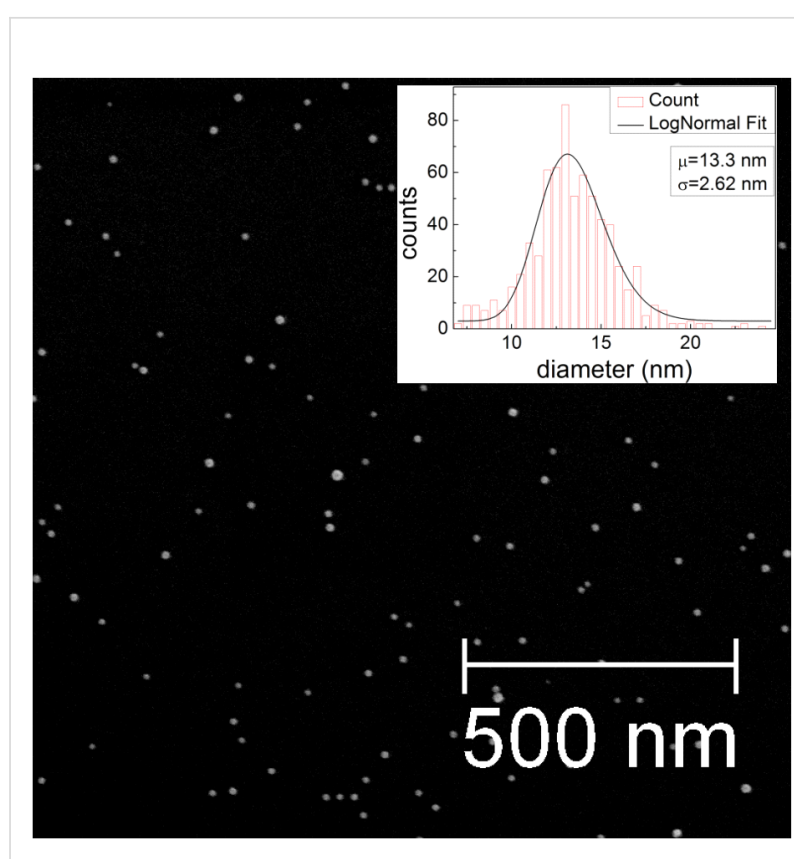

Figure 1: Scanning electron micrograph of Fe nanoparticles deposited on Si. The average particle size observed is $13.3 \mathrm{~nm}$.

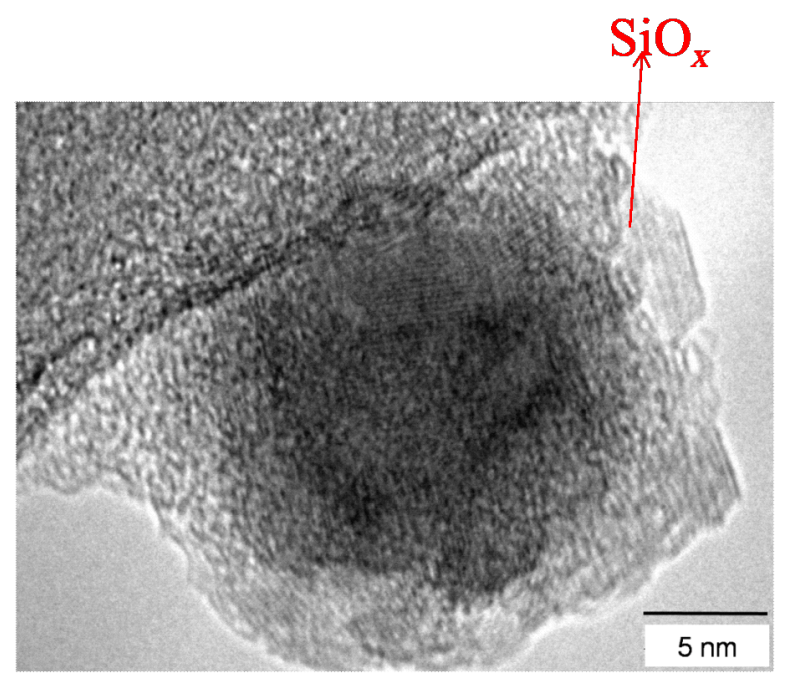

Figure 2: Transmission electron microscope image of Fe nanoparticles (dark contrast) coated with a thin $\mathrm{SiO}_{x}$ layer (brighter contrast).

quite low temperatures if a Pd coating is applied to the surface of the Ta [22]. This leads to changes of the linear dimensions of up to $3 \%$. The actual stress generated in the films depends on the transfer of the elastic strain in the substrate to the particle embedding film. This transfer depends crucially on the interface properties and is also limited by the onset of plastic deformation. Previous computer simulations of Co nanoparticles 


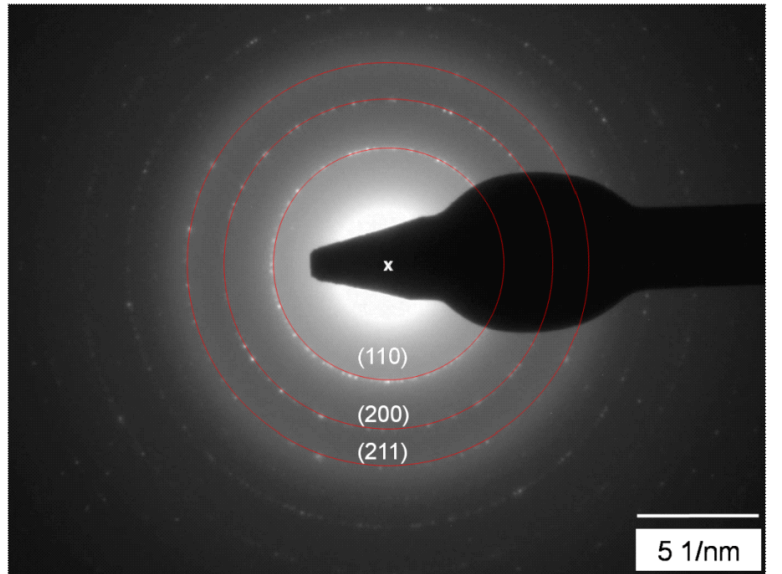

Figure 3: Electron diffraction pattern of the Fe nanoparticles. The Miller indices of the respective lattice planes belong to the bcc structure of $\mathrm{Fe}$.

deposited on a $\mathrm{Cu}$ substrate [23] showed that strain transfer from substrate to particles depends on the structure of the interface, and that the strain is also limited to the part of the nanoparticle adjacent to the interface. To achieve large and uniform elastic deformation of the nanoparticles, it is more appropriate to embed them into a continuous film. In addition, an adhesion layer of Ta has previously been used to improve the strain transfer [24]. In this study, the Fe nanoparticles were embedded in $\mathrm{Cu}$ films. The procedure consisted of an initial deposition of a $10 \mathrm{~nm}$ thick $\mathrm{Cu}$ base layer on the Ta substrate, followed by deposition of the nanoparticles in a second step. Finally, the particles were capped with a $20 \mathrm{~nm}$ thick $\mathrm{Cu}$ layer. This procedure was repeated twice, in order to achieve a higher magnetic signal, resulting in a total $\mathrm{Cu}$ film thickness of $50 \mathrm{~nm}$ (see Experimental section). The $\mathrm{Cu}$ films may also protect the Fe particles from oxidation if exposed to atmospheric conditions. The solubility of $\mathrm{H}$ in $\mathrm{Cu}$ at equilibrium is very low [25], so we did not expect any effects from $\mathrm{H}$ dissolution in the $\mathrm{Cu}$ films during the loading procedure. Another advantage of using $\mathrm{a} \mathrm{Cu}$ film is that the strain in the film can be directly measured by X-ray diffraction, which would be difficult for the $\mathrm{Fe}$ nanoparticles alone due to the low scattering intensity. The stress in the $\mathrm{Cu}$ film was determined in standard Bragg-Brentano geometry, where the in-plane stress can be calculated from the measured variation of the interplanar distance of the lattice planes parallel to the film plane. For calculation of the stress, an average elastic constant of the $\mathrm{Cu}$ film and the embedded Fe nanoparticles should be used. However, since the volume fraction of the Fe nanoparticles was only $0.04 \%$, the average elastic modulus of the film with the nanoparticles was practically identical to the modulus of $\mathrm{Cu}, E_{\mathrm{Cu}}=110 \mathrm{GPa}$.
The samples were loaded under a hydrogen pressure of 2 bar at a temperature of $100{ }^{\circ} \mathrm{C}$ for durations of up to several hours. After each loading cycle, the lattice parameters of the Ta and the $\mathrm{Cu}$ were determined by X-ray diffraction. The volume expansion of the Ta foils led to a shift of the Ta Bragg reflections to lower diffraction angles, while at the same time the Poisson contraction of the $\mathrm{Cu}$ film in the direction perpendicular to the film plane resulted in a shift of the $\mathrm{Cu}(111)$ fcc peak to higher angles. Both effects can be observed in Figure 4, in which X-ray diffraction patterns of the samples in the as-prepared state and after $24 \mathrm{~h}$ exposure to hydrogen gas are shown. The presence of a strong $\mathrm{Cu}(111)$ reflection indicates textured growth of the $\mathrm{Cu}$ layer with the close packed planes parallel to the substrate surface. The $\mathrm{Ta}(110)$ peak shifts from $2 \Theta=38.51^{\circ}$ to $38.20^{\circ}$. This corresponds to an expansion by $0.78 \%$ in the direction perpendicular to the surface. Since the Ta foil is much thicker than the $\mathrm{Cu}$ film, the expansion of the Ta foil occurs in the same way in the plane of the foil. The $\mathrm{Cu}$ film has to follow this expansion, which reflects itself in a contraction perpendicular to the film surface [21]. The observed shift of the $\mathrm{Cu}(111)$ peak from $2 \Theta=43.383^{\circ}$ to $43.421^{\circ}$ corresponds to a contraction of $\varepsilon_{\mathrm{Z}}=-0.083 \%$. Applying Hooke's law for elastically isotropic media, this leads to an in-plane strain of $\varepsilon_{\mathrm{X}}=0.09 \%$ in the $\mathrm{Cu}$ film. The biaxial stress can then be calculated using $\sigma=\varepsilon_{\mathrm{X}} E_{\mathrm{Cu}} /(1-v)$, (where $v$ is Poisson's ratio), resulting in a tensile stress value $\sigma=+0.15 \mathrm{GPa}$ for the $\mathrm{Cu}$ film with the embedded Fe nanoparticles. Another result is that the in-plane strain in the $\mathrm{Cu}$ film is lower than the strain in the Ta substrate. This can be a result of plastic deformation inside the $\mathrm{Cu}$ film, or of some sliding processes at the $\mathrm{Ta} / \mathrm{Cu}$ interface leading to incomplete transfer of strain from the Ta to the $\mathrm{Cu}$ film.

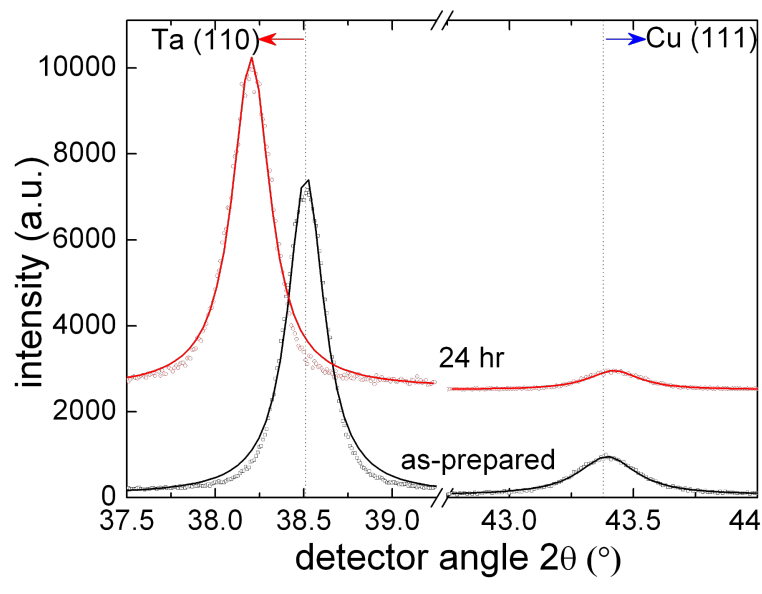

Figure 4: X-ray diffraction patterns (Cu Ka radiation) of Fe nanoparticles embedded in a Cu film on a Ta substrate in the as-prepared state and after $24 \mathrm{~h}$ of loading with hydrogen. The solid lines represent fits to the measured data using Lorentz functions. 


\section{Magnetic properties}

Since the $\mathrm{H}$ remains in the Ta substrates after loading for a long time [9], it is possible to study the effect of stress on the properties of the nanoparticle by standard ex-situ techniques after the loading process. To investigate the effect of the applied stress on the magnetic properties of the Fe nanoparticles, measurements in a SQUID magnetometer were performed over a range of temperatures. Figure 5 shows the magnetization curves of the $13 \mathrm{~nm}$ embedded Fe nanoparticles at a temperature $T=10 \mathrm{~K}$; the magnetic field was applied parallel to the plane of the film (in-plane). The paramagnetic signal of the Ta substrate was fitted with a straight line and has been subtracted from the data. Note that the total ferromagnetic moment of $\mathrm{Fe}$ nanoparticles $\left(8.3 \cdot 10^{-6} \mathrm{emu}\right)$ in saturation is only about $2 \%$ of the paramagnetic signal of the Ta substrate at 5000 Oe. At this temperature, the particles show a ferromagnetic behaviour. It should be noted that in the as-prepared state the Fe nanoparticles show a superparamagnetic behaviour in our SQUID measurements at $300 \mathrm{~K}$. A direct comparison of the identical sample before and after hydrogen loading showed an almost identical coercivity, but a significant increase of the $M_{\mathrm{r}} / M_{\mathrm{S}}$ ratio ( $M_{\mathrm{r}}$ is the remanence) from $M_{\mathrm{r}} / M_{\mathrm{S}}=0.11$ in the as-prepared state to $M_{\mathrm{r}} / M_{\mathrm{S}}=0.22$ in the loaded state. In addition, the saturation field $H_{\mathrm{S}}$ decreased from a value of about 4 to $5 \mathrm{kOe}$ in the as-prepared state to about 2.5 to $3 \mathrm{kOe}$ in the loaded state (the determination of more precise values for $H_{\mathrm{S}}$ is hindered by the scatter in the measured magnetization data). Both observations indicate a modification of the total effective magnetic anisotropy $K_{\text {eff }}$ by the H loading procedure.

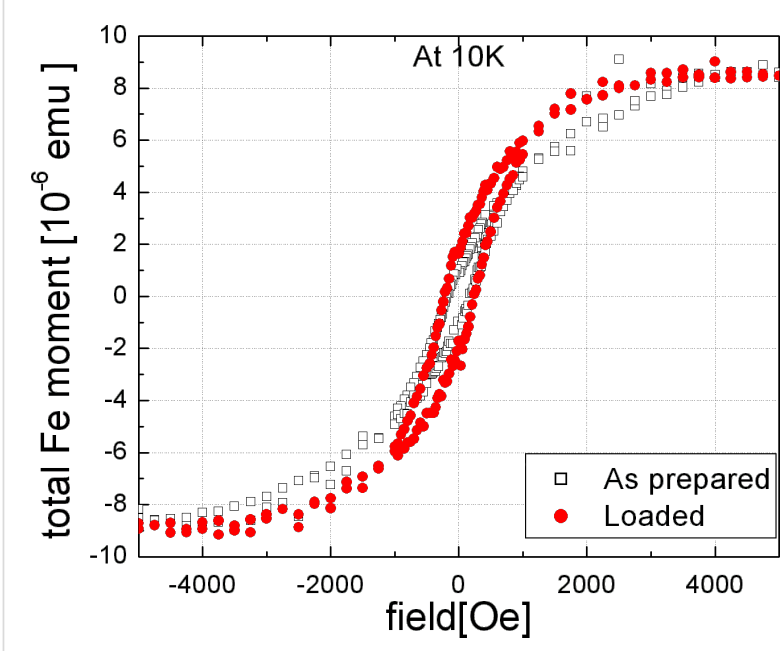

Figure 5: In-plane hysteresis curves of the embedded Fe nanoparticles measured at $10 \mathrm{~K}$ in as-prepared state (open squares) and after loading of the Ta substrate with $\mathrm{H}$ (red circles).

Considering the orientation of the applied field, it appears that the anisotropy has an additional in-plane component after $\mathrm{H}$ loading, which leads to a stronger tendency of the magnetization to align in the plane of the film. This can explain both the larger $M_{\mathrm{r}} / M_{\mathrm{S}}$ ratio (since the component of $M$ parallel to the film plane is measured here) and the reduction of $H_{s}$. It should be noted that the observation of these changes does not contradict the fact that the coercivity does not change. The coercivity depends on the magnetization reversal process which may, in principle, occur by rotation mechanisms restricted to the plane of the film, since the external field is applied parallel to it. In this case one would not expect to see much effect of the additional anisotropy component on the magnetization reversal process, since under the state of biaxial stress there will be no preferential orientation of the magnetization inside each particle towards any specific direction in the plane of the film. However, the fact that $H_{\mathrm{s}}$ changes is a clear indication of a modified magnetic anisotropy, since it directly measures the energy necessary for alignment of the magnetization with the applied magnetic field. From the difference between the hysteresis loops before and after the loading, the contribution of $K_{\text {me }}$ can be estimated to $K_{\mathrm{me}}=4 \cdot 10^{4} \mathrm{~J} / \mathrm{m}^{3}$, which is of similar magnitude as the effects of stress previously observed in $\mathrm{Ni}$ films [10]. For the calculation of $K_{\text {me }}$, we have assumed here that $\mathrm{Fe}$ nanoparticles have the bulk saturation magnetization. We point out that the change of the shape anisotropy due to the elastic deformation of the nanoparticles does not lead to a contribution of comparable size [26].

We have also studied magnetic properties of the embedded Fe nanoparticles at different temperatures. Figure 6 shows the magnetization curves for the loaded sample measured at $10 \mathrm{~K}$ and $300 \mathrm{~K}$. Although a large reduction of the coercivity is observed at $300 \mathrm{~K}$ compared to $10 \mathrm{~K}$, there is a small remnant magnetization indicating that at least some of the particles show ferromagnetic behaviour at $300 \mathrm{~K}$. This may be attributed to the presence of a small fraction of larger Fe nanoparticles (as indicated by the size distribution given in Figure 1). We note here that there may be a small error induced by the subtraction of the large paramagnetic signal of the Ta substrate; therefore, the small difference between the magnetization curves at $300 \mathrm{~K}$ and $10 \mathrm{~K}$ for high applied fields (where the paramagnetic Ta contribution is large) is within the error of the measurement.

One characteristic feature of superparamagnetic behaviour is the existence of a blocking temperature $T_{\mathrm{B}}$, below which the magnetic fluctuations are "frozen in" on the time scale of the respective measurement. A standard technique to characterize superparamagnetic particles is the comparison of the magnetization versus temperature curves, recorded during heating from low temperature (below $T_{\mathrm{B}}$ ) to room temperature. The measurements are performed after previously cooling with applied field (field cooled, FC) or without an external applied magnetic field 


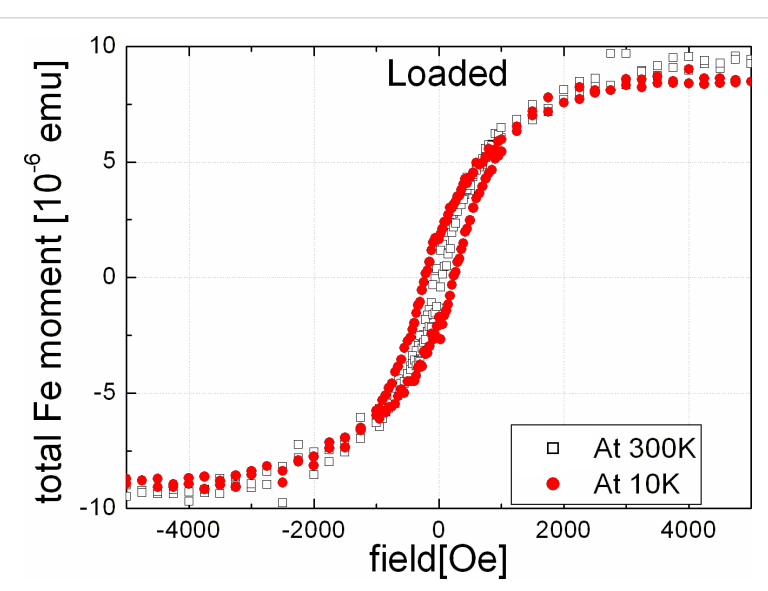

Figure 6: In-plane hysteresis curves of the embedded Fe nanoparticles after loading of the Ta substrate with $\mathrm{H}$, measured at $10 \mathrm{~K}$ (filled red circles) or $300 \mathrm{~K}$ (open squares).

(zero field cooled, ZFC). The thermo-magnetic curves are typically measured in a small applied field. In this study, we applied a field of 100 Oe during the measurements.

Figure 7 shows a comparison of the FC and ZFC curves of the same sample before and after $\mathrm{H}$ loading of the Ta substrate. The ZFC curves show a very similar behaviour before and after loading, whereas the FC curves differ for both samples. As a result, the temperature above which the FC and the ZFC curves fall together (called convergence temperature $T_{\text {con }}$ here) shifts from about $T_{\text {con }}=170 \mathrm{~K}$ in the as-prepared state to about $T_{\text {con }}=220 \mathrm{~K}$ after $\mathrm{H}$ loading. In fact, we do not necessarily expect to see a shift of the blocking temperature $T_{\mathrm{B}}$ (determined by the maxima of the ZFC curves) as a result of an additional magneto-elastic anisotropy contribution. As in the static magnetization measurements, we measure only the magnetization component in the direction of the applied field, which is in the plane of the film here. The fact that $T_{\mathrm{B}}$ does not change indicates that we do not "trap" the magnetization in local minima separated by a barrier. Under the applied biaxial stress, the magneto-elastic anisotropy contribution will add to the other anisotropies present, and we will only see an effect on $T_{\mathrm{B}}$ if the result would be such a "trap state". However, since there is no preferred easy axis direction generated by the biaxial stress in the case of materials with positive magnetostriction, it is not at all evident that we should get such a state. The fact that $T_{\mathrm{B}}$ does not change indicates that the magnetization may fluctuate in the plane of the film in the same way as before the $\mathrm{H}$ loading. In terms of the "energy landscape" picture often used to describe the onset of superparamagnetism, this would translate to a shift of the whole landscape to a lower value without modification of the peak-to-valley differences (as far as only in-plane rotations of the magnetization are concerned). It does not, however, contradict the observed changes of $H_{\mathrm{S}}$ and the $M_{\mathrm{r}} / M_{\mathrm{S}}$ ratio which result from a preferred orientation of the magnetization in the plane of the film without any special easy axis direction.

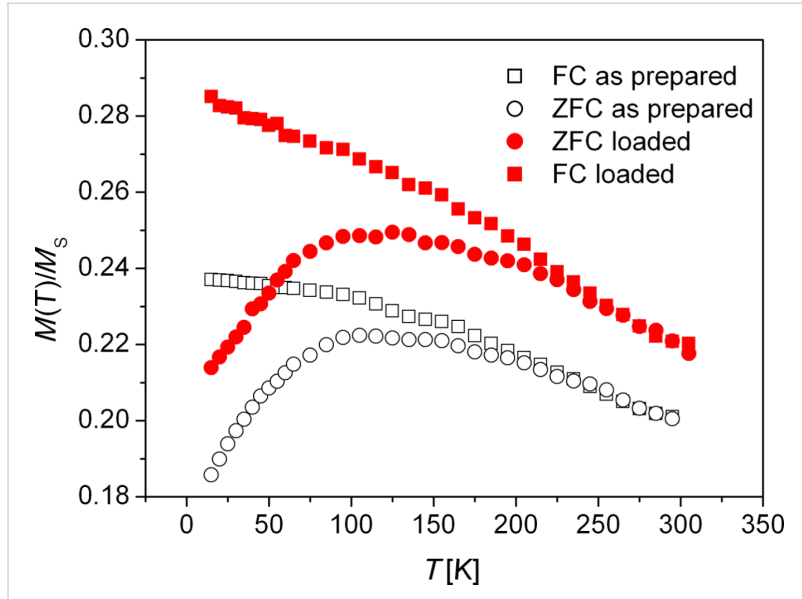

Figure 7: ZFC (circles) and FC (squares) magnetization curves of the $\mathrm{Fe}$ nanoparticles embedded in $\mathrm{Cu}$ film in the as-prepared state (open symbols) and after loading of the Ta substrate with $\mathrm{H}$ (filled red symbols) at $H=100 \mathrm{Oe}$. The magnetization values have been normalized to the saturation magnetization $M_{\mathrm{s}}$ at $10 \mathrm{~K}$.

If we apply the standard criterion $25 k_{\mathrm{B}} T_{\mathrm{B}}=K_{\text {eff }} \cdot V$ also as a rough estimate for the effect on $T_{\text {con, }}$, then a shift of $50 \mathrm{~K}$ in $T_{\text {con }}$ would correspond to an increase of the effective anisotropy energy density $K_{\text {eff }}$ by approximately $1.5 \cdot 10^{4} \mathrm{~J} / \mathrm{m}^{3}$ for $13 \mathrm{~nm}$ diameter particles. This is similar to the contribution from $K_{\mathrm{me}}$ estimated from the hysteresis curves above. One may use a simple estimation of the magneto-elastic energy in the form $K_{\mathrm{me}}=3 / 2 \cdot \sigma \cdot \lambda$, where $\lambda$ is the magnetostriction constant of the material. Using $\lambda=8 \cdot 10^{-6}$ as an estimate for Fe [27], the observed change of $K_{\text {eff }}$ would correspond to a stress of about $1 \mathrm{GPa}$, which is larger than the average stress in the $\mathrm{Cu}$ film as estimated from the X-ray diffraction experiments. It is unclear at the moment whether this is due to a deviation of the local stress value in the Fe nanoparticles from the average stress in the $\mathrm{Cu}$ film, or due to a deviation of $\lambda$ from the value for bulk Fe.

It is interesting to compare these results with earlier research on Fe nanoparticles. A recent report on the structure, morphology and magnetic properties of Fe nanoparticles deposited on single crystal surfaces can be found in [28]. In earlier studies, Methling et al. [29] observed the onset of superparamagnetism in size-selected Fe clusters at room temperature for sizes below $11 \mathrm{~nm}$. However, the particles studied here, of nominally $13 \mathrm{~nm}$ diameter, behave superparamagnetically in the as-prepared state at room temperature. The difference may result from a slight over estimation of the average size in this study due to the method used (evaluation of SEM images). Such an over estimation could also arise from a non-spherical shape of the nanopar- 
ticles, as has been observed in [30] where a height-to-width ratio of 0.85 was found for $\mathrm{Fe}$ nanoparticles produced under similar conditions.

Another interesting point is the low value of the $M_{\mathrm{r}} / M_{\mathrm{S}}$ ratio observed for our Fe nanoparticles. According to the Stoner-Wohlfarth model, a value of $M_{\mathrm{r}} / M_{\mathrm{S}}=0.5$ would be expected for a random distribution of the easy axis for particles with uniaxial anisotropy. For the Fe nanoparticles investigated here, a cubic anisotropy is expected which would further increase the ratio of $M_{\mathrm{r}} / M_{\mathrm{S}}$. The low value found here could result from dipolar interactions between particles stacked vertically above each other (which might occur during the deposition of the second layer of Fe nanoparticles). Dipolar interaction can also influence the dynamic behavior of the magnetization [31]. In addition, the Fe nanoparticles may also experience Ruderman-Kittel-Kasuya-Yosida (RKKY) like coupling through the $\mathrm{Cu}$ matrix, which depends on the details of the arrangement of the particles. On the other hand it is well-known that the particle surfaces may lead to additional anisotropies in nanoparticles. For example, in Co nanoparticles this leads to size dependent effective anisotropies [32]. As a result, the spin structure can assume a non-collinear state as a minimum energy configuration, which would also lead to a reduction of the $M_{\mathrm{r}} / M_{\mathrm{S}}$ ratio. A recent study of the properties of individual $\mathrm{Fe}$ nanoparticles by photoemission spectroscopy [33] indicates that, depending on the size of the nanoparticles, different spin structures may result. It should be mentioned here that the presence of sub-particles with different lattice orientation in our samples may lead to a reduction of the crystalline anisotropy according to the random anisotropy model, which is commonly applied to explain the properties of nanocrystalline soft magnets [34]. This effect could further increase the influence of local surface anisotropies on the local spin structure.

Finally, it is noted that the contribution of $K_{\mathrm{me}}$ to the anisotropy in magnetic nanoparticles may be combined with any of the other contributions as given in Equation 1. It may therefore be useful for the optimization of the magnetic properties of future magnetic data storage media.

\section{Conclusion}

In conclusion, it has been demonstrated that large biaxial stress, as a result of hydrogen loading of the substrate of embedded Fe nanoparticles in the size regime of $13 \mathrm{~nm}$, leads to a modification of their magnetic properties. Results of static magnetization measurements have been presented showing large increases of the $M_{\mathrm{r}} / M_{\mathrm{S}}$ ratio and a reduction of the saturation field of the nanoparticles. The temperature dependent magnetization curves obtained after field cooling are also influenced by the applied stress. The results may be explained by an additional magneto- elastic anisotropy which leads to an "easy plane" rather than an "easy axis". The results may be useful for the optimization of the magnetic properties of future magnetic data storage media.

\section{Experimental}

Iron (Fe) nanoparticles were generated using a custom-built plasma gas condensation (PGC) chamber. Iron metal "vapour" was generated using a 2" magnetron sputter source (MAK II) which was loaded with a $99.95 \%$ pure Fe target. A continuous argon (Ar) gas stream, adjusted using a mass flow controller, was used as the sputtering gas source and also acts as a condensation gas. A constant Ar pressure of 0.85 mbar was maintained in the PGC chamber for generating Fe nanoparticles. At this Ar pressure iron metal vapour, generated at a sputtering power of $30 \mathrm{~W}$, condenses in to small nuclei, which grow further by adding Fe atoms or cluster-cluster aggregation. The distance between the first aperture in the PGC chamber and the sputter source (called the aggregation length) influences the particle size. In this study, an aggregation length of $170 \mathrm{~mm}$ was used. Nanoparticles formed in the PGC chamber were transported to the deposition chamber by maintaining a lower pressure in the deposition chamber compared to PGC chamber. The base pressure of the deposition chamber was lower than $1 \cdot 10^{-7}$ mbar. Using another $4 "$ sputter source and a thermal evaporator, both located inside the deposition chamber, thin films could be deposited on the substrate simultaneously with the Fe particles. In this way, embedding of the Fe nanoparticles on the chosen substrate into a protective film could be achieved. For structural analysis in the TEM, Fe nanoparticles were deposited on copper TEM grids covered with holey carbon films, and subsequently covered by deposition of a $5 \mathrm{~nm}$ thick $\mathrm{SiO}_{x}$ film on top using thermal evaporation. Ta foil of $200 \mu \mathrm{m}$ thickness was used as a substrate for the stress application. One side of the Ta foil was polished to an RMS roughness of less than $5 \mathrm{~nm}$ using chemo-mechanical polishing. The other side of the Ta foil was coated with $100 \mathrm{~nm}$ of palladium, which acts as a catalyst for the hydrogen loading. Afterwards a $10 \mathrm{~nm}$ thick Ta adhesion layer was deposited on the polished side by DC sputtering. On top of the Ta layer, a $10 \mathrm{~nm} \mathrm{Cu}$ layer was thermally evaporated. Fe nanoparticles generated in the PGC chamber were then deposited on the $\mathrm{Cu}$ layer for $300 \mathrm{~s}$. After deposition, the Fe nanoparticles were covered with a $20 \mathrm{~nm}$ thick $\mathrm{Cu}$ layer. To get a reasonably large magnetic signal for the magnetic measurements, another set of Fe nanoparticles was deposited using the same parameters as before. Finally, a $20 \mathrm{~nm}$ thick $\mathrm{Cu}$ layer was deposited as a protective cover.

\section{Acknowledgements}

Financial support by the Baden-Württemberg Stiftung in the framework of the "Functional Nanostructures" program is gratefully acknowledged. 


\section{References}

1. Stoner, E. C.; Wohlfarth, P. Philos. Trans. R. Soc. London A 1948, 240 599-642. doi:10.1098/rsta.1948.0007

2. Néel, L. Ann. Geophys. (C. N. R. S.) 1949, 5, 99-136.

3. Brown, W. F., Jr. Phys. Rev. 1963, 130, 1677-1686. doi:10.1103/PhysRev.130.1677

4. Weller, D.; Moser, A.; Folks, L.; Best, M. E.; Lee, W.; Toney, M. F.; Schwickert, M.; Thiele, J.-U.; Doerner, M. F. IEEE Trans. Magn. 2000, 36, 10-15. doi:10.1109/20.824418

5. Sun, S.; Murray, C. B.; Weller, D.; Folks, L.; Moser, A. Science 2000, 287, 1989-1992. doi:10.1126/science.287.5460.1989

6. Ethirajan, A.; Wiedwald, U.; Boyen, H.-G.; Kern, B.; Han, L.; Klimmer, A.; Weigl, F.; Kästle, G.; Ziemann, P.; Fauth, K.; Schütz, G.; Jun, C.; Behm, R. J.; Büttner, M.; Romanyuk, A.; Oelhafen, P.; Walther, P.; Biskupek, J.; Kaiser, U. Adv. Mater. 2007, 19, 406-410. doi:10.1002/adma.200601759

7. Skumryev, V.; Stoyanov, S.; Zhang, Y.; Hadjipanayis, G.; Givord, D.; Nogues, J. Nature 2003, 423, 850-853. doi:10.1038/nature01687

8. Nogues, J.; Sort, J.; Langlais, V.; Doppiu, S.; Dieny, B.; Munoz, J. S.; Surinach, S.; Baro, B. D.; Stoyanov, S.; Zhang, Y. Int. J. Nanotechnol. 2005, 2, 23-42.

9. Reiss, G.; Huetten, A. Nat. Mater. 2005, 4, 725-726. doi:10.1038/nmat1494

10. Hamann, J. E.; Mohanan, S.; Herr, U. J. Appl. Phys. 2007, 102, 113910. doi:10.1063/1.2821309

11. Mohanan, S.; Herr, U. J. Appl. Phys. 2007, 102, No. 093903. doi:10.1063/1.2802993

12. Andersson, G.; Burkert, T.; Warnicke, P.; Björck, M.; Sanyal, B.; Chacon, C.; Zlotea, C.; Nordström, L.; Nordblad, P.; Erikson, O. Phys. Rev. Lett. 2006, 96, 037205. doi:10.1103/PhysRevLett.96.037205

13. Winkelmann, A.; Przybylski, M.; Luo, F.; Shi, Y.; Barthel, J. Phys. Rev. Lett. 2006, 96, 257205. doi:10.1103/PhysRevLett.96.257205

14. Wegner, K.; Piseri, P.; Tafreshi, H. V.; Milani, P. J. Phys. D: Appl. Phys. 2006, 39, R439-R459. doi:10.1088/0022-3727/39/22/R02

15. Hihara, T.; Sumiyama, K. J. Appl. Phys. 1998, 84, 5270-5276. doi:10.1063/1.368776

16. Rubin, S.; Holdenried, M.; Micklitz, H. Eur. Phys. J. B 1998, 5, 23-28. doi:10.1007/s100510050414

17. Serrano-Guisan, S.; Di Domenicantonio, G.; Abid, M.; Abid, J.; Hillenkamp, M.; Gravier, L.; Ansermet, J.; Felix, C. Nat. Mater. 2006, 5, 730-734. doi:10.1038/nmat1713

18. Rui, X.; Sun, Z. G.; Xu, Y.; Sellmyer, D. J.; Shield, J. E. J. Appl. Phys. 2005, 97, No. 10K310. doi:10.1063/1.1853271

19. Terheiden, A.; Rellinghaus, B.; Stappert, S.; Acet, M.; Mayer, C. J. Chem. Phys. 2004, 121, 510-516. doi:10.1063/1.1760077

20. Granqvist, C. G.; Buhrman, R. A. J. Appl. Phys. 1976, 47, 2200-2219. doi:10.1063/1.322870

21. Mohanan, S.; Smetanin, M.; Weissmüller, J.; Herr, U. Scr. Mater. 2009, 60, 756-759. doi:10.1016/j.scriptamat.2009.01.005

22. Pick, M. A.; Davenport, J. W.; Strongin, M.; Dienes, G. J. Phys. Rev. Lett. 1979, 43, 286-289. doi:10.1103/PhysRevLett.43.286

23. Zhou, J.; Saranu, S.; Herr, U. J. Phys.: Conf. Ser. 2009, 144, 012010. doi:10.1088/1742-6596/144/1/012010

24. Remhof, A.; Song, G.; Sutter, C.; Schreyer, A.; Siebrecht, R.; Zabel, H.; Güthoff, F.; Windgasse, J. Phys. Rev. B 1999, 59, 6689-6699. doi:10.1103/PhysRevB.59.6689

25. Massalski, T. B., Ed. Binary alloy phase diagrams; ASM International: Materials Park, Ohio, 1990.
26. Osborn, J. A. Phys. Rev. 1945, 67, 351-357. doi:10.1103/PhysRev.67.351

27. Chen, C.-W. Magnetism and Metallurgy of Soft Magnetic Materials; Dover Publications: New York, 1986.

28. Kleibert, A.; Rosellen, W.; Getzlaff, M.; Bansmann, J. Beilstein J. Nanotechnol. 2011, 2, 47-56. doi:10.3762/bjnano.2.6

29. Methling, R.-P.; Senz, V.; Klinkenberg, E.-D.; Diederich, T.; Tiggesbäumker, J.; Holzhüter, G.; Bansmann, J.; Meiwes-Broer, K. H. Eur. Phys. J. D 2001, 16, 173-176. doi:10.1007/s100530170085

30. Kleibert, A.; Bulut, F.; Gebhardt, R. K.; Rosellen, W.; Sudfeld, D.; Passig, J.; Bansmann, J.; Meiwes-Broer, K. H.; Getzlaff, M. J. Phys.: Condens. Matter 2008, 20, 445005. doi:10.1088/0953-8984/20/44/445005

31. Majetich, S. A.; Sachan, M. J. Phys. D: Appl. Phys. 2006, 39, R407-R422. doi:10.1088/0022-3727/39/21/R02

32. Respau, M.; Broto, J. M.; Rakoto, H.; Fert, A. R.; Thomas, L.; Barbara, B.; Verelst, M.; Snoeck, E.; Lecante, P.; Mosset, A.; Osuna, J.; Ould Ely, T.; Amiens, C.; Chaudret, B. Phys. Rev. B 1998, 57, 2925-2935. doi:10.1103/PhysRevB.57.2925

33. Fraile Rodriguez, A.; Kleibert, A.; Bansmann, J.; Voitkans, A.; Heyderman, L.; Nolting, F. Phys. Rev. Lett. 2010, 104, 127201. doi:10.1103/PhysRevLett.104.127201

34. Herzer, G. IEEE Trans. Magn. 1990, 26, 1397-1402. doi:10.1109/20.104389

\section{License and Terms}

This is an Open Access article under the terms of the Creative Commons Attribution License (http://creativecommons.org/licenses/by/2.0), which permits unrestricted use, distribution, and reproduction in any medium, provided the original work is properly cited.

The license is subject to the Beilstein Journal of

Nanotechnology terms and conditions:

(http://www.beilstein-journals.org/bjnano)

The definitive version of this article is the electronic one which can be found at: doi:10.3762/bjnano.2.31 